\title{
Organise a Clinical Trial
}

\author{
JP Garner
}

\section{Introduction}

Clinical trials take many forms depending upon the hypothesis being examined but they all follow a similar general framework, parts of which are alluded to in this article. The one factor that remains constant throughout any clinical trial is the amount of work involved in its initiation and running and it is of paramount importance that this is not underestimated when beginning to plan. Trials falter and fail for many reasons, but the commonest is probably that those running it no longer have time to attend to it, and untended a trial will wither and die.

One can give a trial its best chance of success by taking time in the preparatory phases to think carefully through exactly what is going to be involved in the trial and planning for it ahead of time. This short article will provide a starting point from which to begin, and some pointers as to what needs to be considered. It is not an authoritative guide and many things will need to be checked locally to comply with regional policy regarding clinical trials.

\section{Have An Idea}

This is an essential step and is often the most difficult. Most of us find original thought difficult and so coming up with a reasonable idea that is worth trialling can prove frustrating. It may well be that it is ones boss that has the idea, either in one of their regular 'flash of genius' moments or a long cherished pet theory that they have never been able to prove. In either event it is essential to sit down and examine the proposed trial idea from every angle. Has that idea been looked at before? Is it worthwhile looking at such an idea or is it simply another candidate for the 'too hard' pile? A proposal should not be discarded simply because it appears too hard, but it is essential to look for the balance between the amount of work likely to be needed to see such a project through to fruition and the potential gains both clinically and personally. There is no point devoting 2 years work to an idea that won't change clinical practice and is unlikely to be publishable at the end.

\section{Literature Search}

This is perhaps the most important aspect of the whole preparatory phase. A diligent and thorough review of the literature is likely to be helpful on many counts. If the work has already been done and the planned hypothes is either proven or disproven in a scientifically valid trial then further efforts are likely to be nugatory. The drug or treatment under consideration may have been used in a similar, but not identical, fashion elsewhere and those trial papers might give several useful pointers towards trial organisation, endpoints or numbers. They will also serve as a justification as to why it is worth undertaking a trial. Finally, a literature search will furnish a body of evidence about current practice. This too forms part of the justification in showing the intention to demonstrate advantage, of whatever form, over current best practice; it will also form the basis for the discussion section of the paper when the results are published. It is generally insufficient to rely on abstracts alone and every effort should be made to obtain - and then read - the original papers. In performing a literature search an idea will also be gained of which journals are likely to be interested in the finished paper, by seeing who has published similar articles.

\section{General Trial Organisation}

The decision must be made at the outset as to the general type of trial to be undertaken. Will the trial be randomised or not? Blinded, double-blinded or open? These are concepts that may confuse the newcomer and Box 1 explains these terms. Having decided upon the general format of the trial the next step is to begin working through the development of a trial protocol. This details exactly what happens along the way from recruitment (or even before recruitment) of the patient to achievement of the clinical endpoint and the requirements for a complete trial protocol are given in Box 2. It requires detailed instructions as to what should happen at every given decision point and probably the most easily understood way to do this is with a 'treatment algorithm'. Repeatedly thinking ones way along the protocol pathway will inevitably turn up problems and questions that were not envisaged at the outset, but now is the time to address them as protocol amendments mid-trial are difficult to implement and weaken the scientific validity of the work. This process is also 


\section{Randomisation}

Patients are randomly allocated between treatment (or no treatment) groups to compensate for variations in, and therefore possible bias from, underlying variables other than those being specifically examined. Randomisation can be achieved by using random number tables available in scientific textbooks or generated by computer to determine into which treatment group an individual is placed.

\section{Blinding}

A blinded study is one in which the participant does not know whether they received the treatment under test or the control treatment. A double blind study means that neither the patient nor the researcher knows which

treatment has been allocated. Double-blind studies are the norm in trialling medical therapy but are impossible when examining surgical techniques as the surgeon cannot be blinded to the method of surgery! An open study is one in which participant and researcher both know the treatment allocation. Blinding is a further attempt to minimise bias.

\section{Control Groups}

These form a group against which your treatment group may be compared. They may receive inert treatment, that is placebo, or the current standard therapeutic regimen available. Alternatively they may receive no treatment at all to compare whether intervention in a particular instance is better than no intervention.

\section{Parallel / Crossover Groups}

Participants are allocated to one of a number (n) of treatment groups being used to compare ' $n$ ' different treatments. Comparisons are then made between patients. Alternatively, all patients receive all ' $n$ ' treatments in a predetermined sequence and the effects noted down on each different therapy; comparison is then made of the effect within patients. The benefits of such crossover designs are that inter-patient variability is reduced and the number of participants required is lessened.

\section{Prospective / Retrospective Studies}

Most clinical trials will be prospective i.e. the data set required is determined at the outset and recorded as the trial progresses. Retrospective studies utilise data already collected, which incurs the potential for inadequate data collection.

\section{Intention to Treat}

It is recognised that some participants in a trial may not conform to the trial protocol, or may at a later date be found to actually fulfil an exclusion criteria. Such protocol deviations may bias the results; similar withdrawing many patients from analysis on the basis of protocol deviation may also skew data. Consequently it is normal to perform statistical analysis on an 'intention to treat' basis i.e. as the randomisation procedure dictated, although further subsidiary 'per protocol' analyses may sometimes be undertaken. useful in identifying the personnel who will be involved in the trial. This includes not only medical staff, but also nursing, pharmacy and community health professionals too. If the trial is going to increase either their departmental costs or workload it is politic to consult them in the preparatory phase of the trial and to ask permission. Imposition of extra work without consultation may result in animosity and in such circumstances the aggrieved parties are unlikely to contribute fully and reliably to your study. If the trial will involve the patients of other consultants than one's own, it is again polite to ask permission beforehand.

Box 2. The specific areas that must be addressed when organising a clinical trial.

$$
\begin{aligned}
& \text { - Background and General Aims } \\
& \text { - Specific Objectives } \\
& \text { - Patient Selection Criteria } \\
& \text { - Treatment Schedules } \\
& \text { - Evaluation of Response } \\
& \text { - Trial Design } \\
& \text { - Plans for Statistical Analysis } \\
& \text { - Randomisation of Patients } \\
& \text { - Patient Consent } \\
& \text { - Size of Trial } \\
& \text { - Monitoring Trial Progress } \\
& \text { - Forms and Data Handling } \\
& \text { - Protocol Deviations } \\
& \text { - Administrative Responsibilities }
\end{aligned}
$$

\section{Statistics}

Statistics is a word almost uniformly guaranteed to strike fear into the heart of clinicians but is an unavoidable evil where clinical trials are concerned. Not only is it mandatory to statistically analyse the data at the end of the study but a power calculation at the start is an essential prerequisite [see Box 3]. A certain degree of realism is required when performing power calculations both in defining the level of effect that is deemed significant and in assessing the number of patients the calculation indicates are required. When considering the level of effect of the treatment, historical data is essential for comparison and perusal of similar trials may serve as a guide. The number of patients required to adequately power the study is crucial; any shortfall will weaken the conclusions irreparably and will be found out by the journals to which the paper is submitted, as well as by their readership. It is essential to assess the numbers required against the throughput of suitable patients in the unit; in order to recruit them within a reasonable time frame it may be necessary to undertake the trial in several centres - a multicentre study. It is important to be warned - multicentre studies take even more work to organise and run. Unless one is a talented amateur statistician it is probably worthwhile taking independent professional advice before commencing the trial. Nothing annoys statisticians more than being presented with a bagful of data at the end of the study and being asked to prove something from it; 
knowing what tests will need to be applied, the constraints that this may entail and the numbers needed ahead of time simplifies matters and again adds to the scientific robustness of the work. The trial protocol should include details of which statistical tests one intends to use to analyse the data. Implicit in all of this is the setting of clearly identifiable clinical endpoints that are attainable and reproducible.

Box 3. Calculation of Statistical Power

Power = $1-($ Probability of a Type II error $)$

A type II error describes the case where there is actually a difference between treatments, but this is not detected by the test i.e. a false negative. The power of a study depends on the size of the difference between treatments as well as the sample size. If by the constraints of the study the

sample size must be small then a study will only be

sufficiently powered to detect large differences in

treatment effect. Conversely, a very large sample will allow

identification of small differences in treatment effect.

Standard formulae are available for calculation of power.

\section{Inclusions and Exclusions}

This may seem rather self-evident but can be overlooked. Before any trial can commence it is important to know who the study population is and how prevalent that population is. This is all inextricably linked to the power calculation outlined above and the potential need for a multicentre trial; individual centres can rarely amass enough patients who have rare conditions within a sensible time frame. Just as important as the inclusions, is who to exclude. There will inevitably be patients who are deemed unsuitable for inclusion and these must be specifically detailed at the outset. Inadvertent inclusion of unsuitable participants will skew the data, invalidate the analyses and weaken the conclusions. Previous similar published trials will give useful indications.

\section{Ethics}

It is imperative that the trial conforms to acceptable ethical standards and there will be several hoops to jump through to ensure this. It is usual for all proposed trials to be assessed by the hospital's research and development committee or ethics committee if there is one. After satisfying the hospital committee, the protocol will then have to be presented to the local health authority's regional ethics committee. This will involve provision of a complete protocol and evidence of statistical review. One will be expected to attend an ethical review meeting and answer questions on the trial proposal. One should be prepared for some tough questions and accept that there may be the need for amendments. The written permission of this committee is a requirement of many journals before publication of the finished paper can proceed. The committee also has the power to impose conditions on the trial, the commonest of which is the insistence on an interim report. This may be delivered at the halfway point of the trial in terms of time or when half the expected participants have been recruited. An interim report is not a problem in itself, but one should be aware of the effect interim analyses may have on the overall statistical analysis at the end of the study .

\section{Patient Consent}

Any patient who agrees to participate in a clinical trial must do so of their own free will and without coercion. They must be furnished with sufficient information, in a form that they can understand to, allow them to voluntarily give informed consent. The signed consent form should be kept as a record throughout the trial. It falls to the trial organiser to design both a patient information sheet and a consent form.

\section{Practicalities}

It is imperative to consider exactly what data and parameters are to be collected and precisely how. If questionnaires or scoring systems are to be used, one should ensure that a validated system is chosen; inventing a new one leaves one open to accusations of reaching invalid conclusions. Data collection sheets should be designed that are easy to complete and will also allow easy data transfer onto a spreadsheet. Whilst it is possible to conduct an entire trial and analyse the results on paper, such a Luddite approach will greatly increase the workload; a venture into computerisation will pay dividends in time saved. Careful consideration should be given to which personnel are involved and who is to do what in the trial arrangements. Most junior medical staff will spend only one year in any particular institution after which they will depart to the highlights of St Elsewheres; at this stage one must decide what is to happen to the trial, which is likely to be barely one or two months into patient recruitment given the length of time it takes to organise. Without a committed, interested, individual to oversee the study in ones absence it may falter or even fail, negating all the preceding hard work.

\section{Summary}

Organising a clinical trial is a major undertaking involving a lot of hard work before during and after the study takes place and such trials are one endeavour where there are no short cuts. Cutting corners in an effort to save time, money or effort is usually quite obvious to the assiduous journal reader who will rubbish the conclusions and tarnish one's reputation for scientific validity. By taking time to carefully think through each stage and 
possible eventuality of ones plans it is possible to avoid many of the stumbling blocks that interfere with the conduct of a successful trial. Consideration of the contents of this article may help to clarify matters when organising ones first clinical trial or one may wish to attend one of a growing number of courses that give instruction in many of these matters. 